\title{
Phase structure of self-gravitating systems
}

\author{
Victor Laliena \\ Departamento de Física Teórica, Universidad de Zaragoza, \\ Pedro Cerbuna 12, E-50009 Zaragoza (Spain) \\ laliena@unizar.es
}

January 16, 2003

\begin{abstract}
The equilibrium properties of classical self-gravitating systems in the grand canonical ensemble are studied by using the correspondence with an euclidean field theory with infrared and ultraviolet cutoffs. It is shown that the system develops a first order phase transition between a low and a high density regime. In addition, due to the long range of the gravitational potential, the system is close to criticality within each phase, with the exponents of mean field theory. The coexistence of a sharp first order transition and critical behavior can explain both the presence of voids in large regions of the universe as well as the self-similar density correlations in terms of self-gravity alone.
\end{abstract}

Keywords: Field Theory; Critical Phenomena; Long Range Forces

PACS: 11.10.-z, 64.60.-i, 05.20.-y 


\section{Introduction}

One of the outstanding problems of modern cosmology is the understanding of structure formation in the universe [1. Visible matter at astronomical scales appears organized in a hierarchy of galaxies, cluster and supercluster of galaxies, which tend to be found in filamentary aggregates or two-dimensional sheets that encompass large regions with much lower density of matter and structures. These regions, called voids, occupy a large fraction of the observed universe, and are also organized in a hierarchy [2, 3. The theoretical investigation of void formation is receiving increasingly attention [4].

There is general agreement that the galaxy two point correlation function is scale invariant (self-similar), at least at not too large scales, decaying as a power of the distance, $1 / r^{\gamma}$. The exponent $\gamma$ as well as the scale of homogeneity is still controversial. Pietronero and coworkers found $\gamma \approx 1$ and claim that the power law is obeyed up to the deepest distances [5]. Other authors gave a different exponent $(\gamma \approx 1.8)$ and support the existence of an observed scale of homogeneity [6, 7, 8]. Self-similar behaviour has also been found in the interstellar medium [9, 10, 11, 12.

Although certainly the dynamics must be very important in order to explain these facts, and many physical effects might play a prominent role, it is well possible that many aspects of the observed structures may be understood in terms of the equilibrium states of self-gravitating matter alone, as claimed by the authors of Refs. [13, 14. Indeed, it has been tried to apply thermodynamics to astrophysical systems since a long time (see for instance Ref. [15]). In this paper, we will analyze the general phase structure of a non-relativistic self-gravitating system at thermal equilibrium. The results are general and can be applied to any such system that can be considered to be in these conditions. We will not be concerned here with the very interesting questions of whether thermal equilibrium can be reached in such systems, or the way it is attained. We just assume that the self-gravitating system is at thermal equilibrium.

Let us consider a system of $N$ classical particles of mass $m$ confined in a region of volume $V$ and interacting each other via the Newtonian gravitational potential. Its hamiltonian reads

$$
\mathcal{H}=\frac{1}{2 m} \sum_{i=1}^{N} \boldsymbol{p}_{i}^{2}-\frac{G m^{2}}{2} \sum_{i \neq j} \frac{1}{\left|\boldsymbol{r}_{i}-\boldsymbol{r}_{j}\right|} .
$$

The thermodynamics of such a system is ill defined: the entropy does not exist due to the singularity of the potential at short distances [19, 20]. Furthermore, even if one modifies the potential to remove the short distance singularity, the usual thermodynamical limit does not exist, since the thermodynamic potentials are not extensive, due to the long range gravitational force. In such cases, the microcanonical specific heat can become negative and different statistical ensembles are not equivalent (see Refs. 221, 22, 23, 24, 25. 26, 27, 28, 29, 30]). In particular, the grand canonical ensemble is dominated by completely collapsed configurations, whatever the chemical potential. To use the ordinary thermodynamical tools, the potential must also be modified at long distances ${ }^{1}$.

The thermodynamics of self-gravitating system has been studied since a long time

\footnotetext{
${ }^{1}$ It has been proposed that a kind of thermodynamical limit for systems with potentials decaying as $1 / r$ could be taken by considering the so called dilute regime [31, but it can be shown that this statement cannot hold 32 .
} 
by confining a finite system on a finite box and using mean field theory. The approach developed in this paper is different: we will describe the thermodynamical properties of a self-gravitating system as the limiting case of a family of well behaved, short range systems, the interactions of which decay with distance more and more slowly, thus resembling more and more the newtonian $1 / r$ potential. For these systems the usual thermodynamical limit, which is considered in this paper, does exist. To carry out this investigation, we will take advantage of the fact that the statistics of a self-gravitating ensemble of particles can be related to an euclidean field theory of a single scalar field [13, 14, in a similar way to the relation between the Coulomb gas and the Sine-Gordon field theory [16, 17, 18. Hence, the remaining of the paper will rely on the techniques of euclidean field theory.

\section{Field theoretical description of a self-gravitating system}

The statistical mechanics of a self-gravitating system can be studied in the usual way if the gravitational potential is regularized at short and long distances. Let us choose as a regularized gravitational potential, denoted by $U_{\mathrm{R}}(r)$, an attractive Yukawa potential, with range $1 / m_{0}$, endowed with a hard core of size $r_{0}$ at short distances: $U_{\mathrm{R}}(r)=-G m^{2} \mathrm{e}^{-m_{0} r} / r$ for $r>r_{0}$ and $U_{\mathrm{R}}(r)=\infty$ for $r<r_{0}$. After integrating out the momenta, the grand canonical partition function for chemical potential $\mu$ and temperature $T$ can be written as

$$
\mathcal{Z}_{\mathrm{GC}}=\sum_{N} \mathrm{e}^{\mu N}\left(\frac{m a^{2}}{2 \pi \beta \hbar^{2}}\right)^{3 N / 2} \overline{\mathcal{Z}}_{N}
$$

where $\beta=1 / k_{B} T, a$ is a constant with units of length, to be specified below, and

$$
\overline{\mathcal{Z}}_{N}=\frac{a^{-3 N}}{N !} \int \prod_{i=1}^{N} d^{3} r_{i} \exp \left\{-\frac{\beta}{2} \sum_{i \neq j} U_{\mathrm{R}}\left(\left|\boldsymbol{r}_{i}-\boldsymbol{r}_{j}\right|\right)\right\} .
$$

The partition function $\overline{\mathcal{Z}}_{N}$ can be approximated by dividing the space volume in $\Lambda=V / a^{3}$ cells of size $a$ (the unit length introduced above), and replacing the integrals by appropriate Riemann sums, which can be reorganized as a sum over cell occupation numbers $S_{i}$, with the index $i$ running from 1 to the number of cells, $\Lambda$. If $a$ is of the order of the hard core size, each cell can be either void or occupied by one particle: $S_{i}=0,1$. This is similar to consider self-gravitating fermions [29]. We get

$$
\overline{\mathcal{Z}}_{N}=\sum_{S_{1}=0}^{1} \ldots \sum_{S_{\Lambda}=0}^{1} \exp \left\{\frac{b^{2}}{2}\left[\sum_{n, n^{\prime}} U_{\mathrm{L}}\left(n-n^{\prime}\right) S_{n} S_{n^{\prime}}-U_{\mathrm{L}}(0) N\right]\right\} \delta\left(\sum_{n} S_{n}-N\right)
$$

where $b^{2}=4 \pi \beta G m^{2}, U_{\mathrm{L}}\left(n-n^{\prime}\right)$ is a proper lattice version of $-U_{\mathrm{R}}\left(\boldsymbol{r}-\boldsymbol{r}^{\prime}\right) /\left(4 \pi G m^{2}\right)$, the factor $1 / N$ ! has been canceled due to the distinguishability of classical particles, and the Kronecker delta takes into account that the number of particles is fixed 33.

Since the Yukawa potential is the Green function of $-\nabla^{2}+m_{0}^{2}$, we can choose its lattice version as the lattice Green function of $-\nabla_{\mathrm{L}}^{2}+m_{0}^{2}$, where $\nabla_{\mathrm{L}}^{2}$ is a discretization of the laplacian:

$$
U_{\mathrm{L}}\left(n-n^{\prime}\right)=\frac{1}{a^{3}}\left(\frac{1}{-\nabla_{\mathrm{L}}^{2}+m_{0}^{2}}\right)_{n, n^{\prime}} .
$$


Using (5) and the Hubbard-Stratonovich formula, we can write the following identity:

$$
\begin{aligned}
& \exp \left\{\frac{b^{2}}{2} \sum_{n, n^{\prime}} U_{\mathrm{L}}\left(n-n^{\prime}\right) S_{n} S_{n^{\prime}}\right\}= \\
& C \int[d \psi] \exp \left\{-\frac{1}{2} \sum_{n, n^{\prime}} a^{3} \psi_{n}\left(-\nabla_{\mathrm{L}}^{2}+m_{0}^{2}\right)_{n n^{\prime}} \psi_{n^{\prime}}+b \sum_{n} \psi_{n} S_{n}\right\},
\end{aligned}
$$

where $C$ is a number independent of $S_{n}$. Plugging Eq. (6) in (4) and the resulting equation for $\overline{\mathcal{Z}}_{N}$ in the expression for $\mathcal{Z}_{\mathrm{GC}}$, and performing the summation over $S_{i}$, we can write the grand canonical partition function of the (regularized) self-gravitating system in terms of a local euclidean field theory with a single scalar field $\psi_{n}$ :

$$
\mathcal{Z}_{\mathrm{GC}}=C \int[d \psi] \exp [-\mathcal{S}]
$$

the action of which is given by

$$
\mathcal{S}=\frac{1}{2} \sum_{n n^{\prime}} a^{3} \psi_{n} \mathcal{D}_{n n^{\prime}} \psi_{n^{\prime}}-\sum_{n} \ln \left(1+g \mathrm{e}^{b \psi_{n}}\right),
$$

where $g=\mathrm{e}^{\mu} \mathrm{e}^{-\frac{b^{2}}{2} U_{\mathrm{L}}(0)}\left(\frac{m a^{2}}{2 \pi \beta \hbar^{2}}\right)^{3 / 2}$ and $\mathcal{D}=-\nabla_{\mathrm{L}}^{2}+m_{0}^{2}$. Notice the unusual form of the action: the interaction term, $\ln \left(1+g \mathrm{e}^{b \psi}\right)$, is unbounded from below. It behaves as $-b \psi$ for $\psi \rightarrow \infty$. The action, however, is bounded from below if $m_{0}^{2}>0$. This reflects the fact that the grand canonical ensemble does not exist for non-extensive (long range and/or purely attractive) systems. The infrared and ultraviolet cutoffs, $m_{0}$ and $a$ respectively, make the system short ranged and repulsive at short distances (hard core of size of the order of $a$ ), and thermodynamics is well defined. In the naive continuum limit, in which $a \rightarrow 0(g \rightarrow 0)$ and the relevant field configurations are smooth, we recover the action of Ref. 13. This continuum action is not bounded from below and hence the corresponding functional integral diverges. Thus, the results of Ref. [13 are at most formal and the conclusions different from ours. The regulators, $a$ and $m_{0}$, play an essential role, although the conclusions, as we will see, are independent of them (provided they are small enough).

The action contains four parameters: the lattice spacing, $a$, which obviously has dimension of length; the inverse of the potential range, $m_{0}$, which has dimension of (length) ${ }^{-1}$; $b$, with dimension of (length) $)^{1 / 2}$; and $g$, which is dimensionless. The field is canonically normalized, so that its dimension is (length) ${ }^{-1 / 2}$. It is convenient to redefine the field such that $\phi_{n}=b \psi_{n}+\ln g$, and to work in terms of the dimensionless quantities $\kappa=a / b^{2}$, $\bar{m}_{0}^{2}=a^{2} m_{0}^{2}$, and $h=1 / 2+\kappa \bar{m}_{0}^{2} \ln g$. Ignoring constant terms, the action reads

$$
\mathcal{S}=\frac{\kappa}{2} \sum_{n n^{\prime}} \phi_{n} \overline{\mathcal{D}}_{n n^{\prime}} \phi_{n^{\prime}}-\sum_{n} \ln \cosh \frac{\phi_{n}}{2}-h \sum_{n} \phi_{n}
$$

where $\overline{\mathcal{D}}=-a^{2} \nabla_{\mathrm{L}}^{2}+\bar{m}_{0}^{2}$. An action of the same form, with $\bar{m}_{0}^{2}=0$, has been obtained in Ref. [14 in a similar way. In that case, however, the parameter $h$ has a different meaning. Notice that for $h=0$ the action (9) has the symmetry $\phi_{n} \rightarrow-\phi_{n}$. Since the term linear 
in $\phi_{n}$ breaks this symmetry, we will have $\langle\phi\rangle>0$ for $h>0$ and $\langle\phi\rangle<0$ for $h<0$. At $h=0$ we will have $\langle\phi\rangle=0$ unless the symmetry is spontaneously broken.

The average of particle and energy densities, $\rho$ and $\epsilon$, can be obtained as derivatives of the grand canonical partition function. This allows to express them in terms of the field $\phi$ in the following way:

$$
\begin{aligned}
\rho & =\frac{1}{V} \sum_{n} \frac{\mathrm{e}^{\phi_{n}}}{1+\mathrm{e}^{\phi_{n}}} \\
\epsilon & =k_{B} T\left[\frac{3}{2} \rho-\frac{1}{2} \frac{1}{V} \sum_{n}\left[\phi_{n}-\ln g-\kappa^{-1} \overline{\mathcal{D}}_{n n}^{-1}\right] \frac{\mathrm{e}^{\phi_{n}}}{1+\mathrm{e}^{\phi_{n}}}\right] .
\end{aligned}
$$

One can easily identify the first contribution to the energy in Eq. (11) as the kinetic energy and the second one as the potential energy. The later has the form $\sum_{n}\left(\phi_{n}-\right.$ $\left.\ln g-\kappa^{-1} \overline{\mathcal{D}}_{n n}^{-1}\right) \rho_{n}$, where $\rho_{n}=\mathrm{e}^{\phi_{n}} /\left(1+\mathrm{e}^{\phi_{n}}\right)$ is the local particle density, what implies that the field $\phi_{n}$ represents, up to an additive constant, $\ln g+\kappa^{-1} \overline{\mathcal{D}}_{n n}^{-1}$, the local gravitational potential $^{2}$.

After some algebraic manipulations, which are outlined in the appendix since they are not completely straightforward, we can write the following exact equation for the average energy, which has the expected form:

$$
\epsilon=k_{B} T\left[\frac{3}{2} \rho-\frac{1}{2} \frac{1}{V} \sum_{n} \sum_{r \neq 0} \kappa^{-1}\left\langle\rho_{n} \overline{\mathcal{D}}_{n, n+r}^{-1} \rho_{n+r}\right\rangle\right] .
$$

In the above equation $\epsilon$ and $\rho$ stand for the average energy and density, respectively. Remember that $\overline{\mathcal{D}}_{n, n+r}^{-1} \approx \mathrm{e}^{-\bar{m}_{0} r} / r$. Notices that the fact that the term with $r=0$ is excluded from the sum over $r$ in Eq. (12) implies that the contact term does not contribute to the energy. This, and the bounds $0<\rho_{n}<1$, are manifestations of the particle hard core.

\section{Phase diagram}

Perturbation theory in euclidean field theory starts by identifying the minimum of the classical action and assuming that the relevant field configurations are small fluctuations around this minimum. For the action (9) this is a good approximation if $\kappa$ is large. We shall argue below that it is indeed a good approximation whatever $\kappa$ if $\bar{m}_{0}^{2}$ is small.

The minimum of the action obviously corresponds to the constant field that minimizes the classical potential

$$
\mathcal{U}=\frac{\kappa \bar{m}_{0}^{2}}{2} \phi^{2}-\ln \cosh \frac{\phi}{2}-h \phi
$$

and, therefore, satisfies the equation

$$
\kappa \bar{m}_{0}^{2} \phi=\frac{1}{2} \tanh \frac{\phi}{2}+h .
$$

\footnotetext{
${ }^{2}$ Note that $\overline{\mathcal{D}}_{n n}^{-1}$ is independent of $n$ due to the translational invariance of the Laplacian.
} 


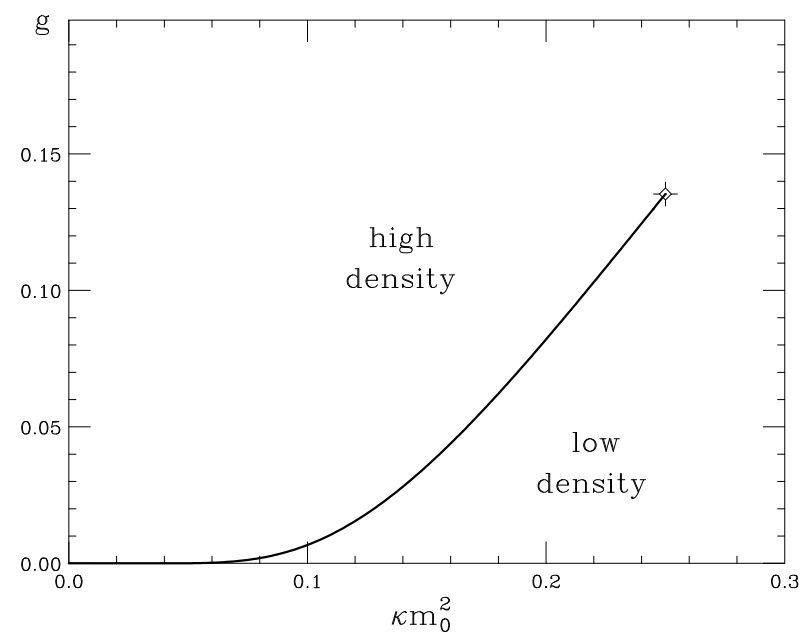

Figure 1: Phase diagram in the $\left(\kappa \bar{m}_{0}^{2}, g\right)$ plane. The solid line is a first order transition that ends at the critical point.

The above equation has one, two or three solutions, depending on the values of $\kappa \bar{m}_{0}^{2}$ and $h$. When it has one solution, it correspond to the global minimum; if there are two solutions, one is the global minimum and the other one a point of inflection; three solutions correspond to a local maximum, a local minimum, and the global minimum. In the last case, the minima can only be degenerate if $h=0$, and then symmetry implies that Eq. (14) has either one or three solutions: for $\kappa \bar{m}_{0}^{2}>1 / 4$ the only solution is $\phi=0$, while for $\kappa \bar{m}_{0}^{2}<1 / 4$ we have two solutions, $\phi= \pm \phi_{0} \neq 0$ (with $\phi_{0}$ positive), which are the two degenerate global minima, besides the symmetric solution $\phi=0$, which is a local maximum. We will denote the two global minima by $\phi_{h}=+\phi_{0}$ and $\phi_{l}=-\phi_{0}$. The densities of each phase are respectively

$$
\rho_{l}=\frac{1}{a^{3}} \frac{\mathrm{e}^{\phi_{l}}}{1+\mathrm{e}^{\phi_{l}}}, \quad \rho_{h}=\frac{1}{a^{3}} \frac{\mathrm{e}^{\phi_{h}}}{1+\mathrm{e}^{\phi_{h}}} .
$$

Hence, the solutions $\phi_{l}$ and $\phi_{h}$ describe phases of low and high density respectively.

The phase diagram in the plane $\left(\kappa \bar{m}_{0}^{2}, g\right)$, at the classical level, is displayed in figure 10 For $\kappa \bar{m}_{0}^{2}>1 / 4$, the transition between the low (small $g$ ) and high (large $g$ ) density regimes is smooth. For $\kappa \bar{m}_{0}^{2}<1 / 4$, the low density phase is separated from the high density phase by a first order transition which takes place at $g_{\mathrm{FO}}=\exp \left[-1 /\left(2 \kappa \bar{m}_{0}^{2}\right)\right]$ (i.e., $h=0)$. This first order line ends at the critical point $\kappa_{c} \bar{m}_{0}^{2}=1 / 4, g_{c}=1 / \mathrm{e}^{2}(h=0)$. The order parameters, $\delta \phi=\phi_{h}-\phi_{l}$ and $\delta \rho=\rho_{h}-\rho_{l}$, vanish at the critical point with the classical (mean field) exponent 1/2. This mean field critical behavior is a consequence of the classical approximation and could be modified by the neglected fluctuations.

For small $\kappa \bar{m}_{0}^{2}$ and $h=0$, Eq. (14) gives $\phi_{h} \approx 1 /\left(2 \kappa \bar{m}_{0}^{2}\right), a^{3} \rho_{h} \approx 1-\mathrm{e}^{-1 /\left(2 \kappa \bar{m}_{0}^{2}\right)}$, $\phi_{l} \approx-1 /\left(2 \kappa \bar{m}_{0}^{2}\right)$, and $a^{3} \rho_{l} \approx \mathrm{e}^{-1 /\left(2 \kappa \bar{m}_{0}^{2}\right)}$. Thus, the low density phase is very dilute, while the high density phase is extremely dense. Most of the densities (including presumably those of physical systems) are between $\rho_{l}$ and $\rho_{h}$, and they correspond, therefore, to the phase coexistence region. 


\section{Critical behavior and correlations}

The corrections to the classical approximation can be obtained perturbatively, by expanding the action around the corresponding minimum. For each phase we write $\phi_{n}=\phi_{h}+\varphi_{n}$ and $\phi_{n}=\phi_{l}+\varphi_{n}$ respectively, and, ignoring again constant terms, the corresponding actions can be written as

$$
\mathcal{S}=\frac{\kappa}{2} \sum_{n n^{\prime}} \varphi_{n} \overline{\mathcal{D}}_{n n^{\prime}} \varphi_{n^{\prime}} \pm \lambda \sum_{n} \varphi_{n}-\sum_{n} \ln \left[1+\lambda\left(\mathrm{e}^{ \pm \varphi_{n}}-1\right)\right]
$$

where the signs + and - correspond to the low and high density phases respectively, and $\lambda=1-a^{3} \rho_{h}=a^{3} \rho_{l}$.

When $\kappa \bar{m}_{0}^{2}$ is very small, $\lambda$ is much smaller and the actions for either phase (16) are very close to the gaussian critical point, $\lambda=0$. Therefore, the classical approximation we are using is very good. In addition, we have a very interesting situation: a very sharp first order transition that separates two phases which, in turn, are close to criticality. Hence, the actions (16) produce a very large correlation length and critical (self-similar) behavior over a vast range of scales. The question is to which universality class corresponds such critical behavior. In three dimensions, the gaussian fixed point is infrared unstable under perturbations of relevant operators. This means that the critical behavior of actions defined in the neighborhood of the gaussian fixed point can be governed by a different (non-gaussian) fixed point. For this to happen, the couplings of the relevant operators of dimension larger than one must be of the order of $\kappa \bar{m}_{0}^{2}$. In our case $\lambda \ll \kappa \bar{m}_{0}^{2}$ and we are in the opposite case: the actions (16) lie very close to the renormalized trajectory of the gaussian fixed point, given by $\lambda=0, \kappa \bar{m}_{0}^{2}>0$. Hence, the critical behavior is governed by the gaussian fixed point, and thus belongs to the mean field universality class.

The correlations can be computed to leading order in $\lambda$ with good accuracy. For the field we get $\left\langle\phi \boldsymbol{r} \phi \boldsymbol{r}^{\prime}\right\rangle-\langle\phi \boldsymbol{r}\rangle\left\langle\phi \boldsymbol{r}^{\prime}\right\rangle=G\left(\boldsymbol{r}-\boldsymbol{r}^{\prime}\right)$, with

$$
G\left(\boldsymbol{r}-\boldsymbol{r}^{\prime}\right)=\int \frac{d^{3} p}{(2 \pi)^{3}} \frac{\mathrm{e}^{\mathrm{i} \boldsymbol{p}\left(\boldsymbol{r}-\boldsymbol{r}^{\prime}\right)}}{p^{2}+\kappa \bar{m}_{0}^{2}},
$$

where we have used the continuum expression since the lattice spacing is much smaller than the correlation length, $\xi=a /\left(\kappa \bar{m}_{0}^{2}\right)^{1 / 2}$. At large distances, $G(\boldsymbol{r})$ decays exponentially as $\exp (-|\boldsymbol{r}| / \xi)$, but in a wide range of distances, $a \ll|\boldsymbol{r}| \ll \xi$, it is approximately self-similar, $G(\boldsymbol{r}) \sim 1 /|\boldsymbol{r}|$.

The correlations of the density (10), $\Gamma\left(\boldsymbol{r}-\boldsymbol{r}^{\prime}\right)$, have the same long distance behavior as the correlations of the field. To leading order in $\lambda$ we have:

$$
\Gamma\left(\boldsymbol{r}-\boldsymbol{r}^{\prime}\right)=\lambda^{2} \mathrm{e}^{G(0)}\left[\mathrm{e}^{G\left(\boldsymbol{r}-\boldsymbol{r}^{\prime}\right)}-1\right] .
$$

For large distances $\Gamma(\boldsymbol{r}) \sim G(\boldsymbol{r})$, and therefore the density correlations behave as $1 /|\boldsymbol{r}|$ for $a \ll|r| \ll \xi$. Hence, the present approach to self-gravitating systems predicts that density correlations decay as a power law with exponent $\gamma=1$ over a vast range of scales. Since $\xi$ is proportional to the assumed range of the gravitational interaction, it is well possible that correlations be self-similar at any observable scale.

It is worthwhile stressing that the above analysis refers to the quasi-critical behaviour of each of the two phases as $\kappa \bar{m}_{0}^{2} \rightarrow 0$. There is a true critical point at $\kappa_{c} \bar{m}_{0}^{2}=1 / 4$ and 
$g=1 / \mathrm{e}^{2}$ (or $\left.\rho_{c}=1 / 2\right)$. In this case the parameters are not small and a non-perturbative analysis is required in order to investigate the nature of its universality class. This critical point might be relevant at very high temperatures, when $\kappa \bar{m}_{0}^{2}$ is of order one.

A similar analysis can be made for small $\kappa$ (strong coupling regime). The symmetry is broken at the classical level if $\kappa \bar{m}_{0}^{2}<1 / 4$. For small $\kappa$ and small $\bar{m}_{0}^{2}$ short wavelength fluctuations cannot induce tunneling between the two minima since it would imply huge fluctuations of the action, of the order $1 / \kappa \bar{m}_{0}^{4}$. Likewise, long wavelength fluctuations cannot induce tunneling since the barrier is too high, of the order of $1 /\left(8 \kappa \bar{m}_{0}^{2}\right)$. The two minima describe phases of low and high density. The correlation length on each phase is of the order of $1 / \bar{m}_{0}^{2}$. Hence, the conclusions are the same as in the weak coupling regime: an abrupt first order transition separates the low and high energy phases, and each phase present critical behaviour of mean field type.

\section{Finite volume effects: the Lane-Emden equation}

It might seem paradoxical that the mean field solution is homogeneous, since it is well known that the usual mean field solutions of self-gravitating systems present density profiles that depend on the distance to the center of the system. There is a simple explanation: since we are looking for the mean field solution of a short ranged translational invariant system in the infinite volume limit it is natural that it be homogeneous. Had we looked for the minimum of the action (8) on a finite box of size $L$ we would have obtained the equation

$$
\sum_{n^{\prime}}\left(-\nabla_{L}^{2}\right)_{n n^{\prime}} \psi_{n^{\prime}}+m_{0}^{2} \psi_{n}-\frac{b g e^{b \psi_{n}}}{1+g e^{b \psi_{n}}}=0 .
$$

On a finite volume, the walls break translational invariance and the solution of Eq. (19) will not be homogeneous, due to the boundary conditions imposed to the operator $\left(-\nabla_{L}^{2}\right)_{n n^{\prime}}$. Since this operator is a discretization of the Laplacian, if $L \ll 1 / m_{0}$, Eq. (19) is similar to the isothermal Lane-Emden equation [34, 35] (if $m_{0}=0$, to leading order in $g$ we will have exactly the discretized Lane-Emden equation, and the corrections in $g$ are due to the cut-off $a$ ), and we will get a solution similar to the known profiles of self-gravitating systems in the mean field approximation. On the other hand, if $L \gg 1 / m_{0}$, the spatial dependence of the solution will be washed out and we will have the reported constant solutions. However, the two solutions correspond to extremely high and extremely low densities, respectively. The system will only be homogeneous at such extreme densities. For intermediate densities, inhomogeneities will necessarily develop: the system will be in a mixture of high and low density domains.

This instability as the volume increases had been notice long ago by Antonov, who found that the solutions of the Lane-Emden equation ceases to be (local) maxima of the entropy if the size of the box is larger than $0.335 G M^{2} /(-E)$, where $M$ is the total mass and $E<0$ the energy, and the system collapses [36]. This was called the gravothermal catastrophe in Ref. 37. 


\section{Final remarks}

In thermal equilibrium, a self-gravitating system is made up of domains of low and high density (voids and clusters). The distribution of domains is a dynamical question that has to do with the way equilibrium is reached. The correlations within each domain are self-similar on a vast range of scales, decaying as $1 / r$. The transition to homogeneity ${ }^{3} 38$ would take place on scales comparable to the range of the gravitational interaction, which may be larger than the deepest observed distances. A similar behavior (first order phase transition and self-similar correlations) was found for the two-dimensional self-gravitating system by using techniques of conformal field theory [39].

It is straightforward to take into account the cosmic expansion in a simplified way, as in Refs. 14, 13, by introducing comoving coordinates $\boldsymbol{x}$ in (11), such that the physical coordinates are $\boldsymbol{r}=R(t) \boldsymbol{x}$, where $R(t)$ is the scale factor. As intuitively expected, this is equivalent to rescale the lattice spacing: $a(t)=R(t) a$ and, consequently, we have the following rescaling of parameters: $\kappa \rightarrow R(t) \kappa$ and $\bar{m}_{0}^{2} \rightarrow R^{2}(t) \bar{m}_{0}^{2}$. This variation of the parameters implies that the difference between the densities of the two phases decreases. This is not surprising: the expansion of the universe acts as a pressure that competes with the tendency of gravity towards collapse.

It is remarkable that the picture of the self-gravitating system devised in this work is strikingly similar to the observed universe, despite it is not at thermal equilibrium. The scaling exponent of the the correlation function of the density, $\gamma=1$, however, is far from the widely accepted exponent of the galaxy correlation function, $\gamma \approx 1.8$ for distances between 0.2 and $20 \mathrm{Mpc}$ [1. As mentioned in the introduction, in the last years there has been a strong debate about the validity of this result. Pietronero and his group analyzed the data from a different perspective and claimed that the exponent of the galaxy correlation function is $\gamma \approx 1$, and that the self-similar behavior extends up to the deepest explored distances. The controversy seems not resolved, although most astrophysicist believe the earlier result, $\gamma \approx 1.8$. This is theoretically supported by the thermodynamic arguments given in [15]. These arguments, however, are somehow heuristic and their validity may be questioned. Indeed, using Eq. (12) as starting point, the same arguments can be applied step by step to the formulation of the self-gravitating system given in this work, leading to the same predictions of Saslaw's book. However, the results of this work, based on a rather rigorous treatment of the self-gravitating system, are completely different.

Hence, the conclusion of this paper is clear: either Pietronero and coworkers are right, and $\gamma \approx 1$, or the thermodynamical approach is not valid to describe the universe at such scales. The later possibility cannot be discarded, since it is difficult to argue that the universe is at thermal equilibrium, and dynamical effects may play a prominent role in the behavior of the galaxy correlation function. In any case, the results obtained in this paper need not be applied to the universe as a whole, or to the large scale structure of it, but to any self-gravitating system that are close to thermal equilibrium. The interstellar gas, where self-similar behavior has also been observed, might be an instance. In this case the scaling exponent of the density correlations is compatible with $\gamma=1$, but with large uncertainties [13.

\footnotetext{
${ }^{3}$ The scale at which the density fluctuations cease to be self-similar.
} 


\section{Acknowledgments}

I thank J.L. Alonso, J.L. Cortés, and A. Galante for useful discussions. This work received financial support from Ministerio de Ciencia y Tecnología (Spain) under the Ramón y Cajal program, and from CICyT (Spain) under the project FPA 2000-1252.

\section{Appendix}

Let us outline in this appendix the derivation of equation (12), which is not completely straightforward. The field $\phi_{n}$ can be obtained from the derivative of the action as

$$
\phi_{n}=\kappa^{-1} \sum_{n^{\prime}} \overline{\mathcal{D}}_{n n^{\prime}}^{-1} \rho_{n^{\prime}}+\kappa^{-1} \sum_{n^{\prime}} \overline{\mathcal{D}}_{n n^{\prime}}^{-1} \frac{\partial \mathcal{S}}{\partial \phi_{n^{\prime}}}-\frac{1 / 2-h}{\kappa \bar{m}_{0}^{2}},
$$

where we used $\sum_{n^{\prime}} \overline{\mathcal{D}}_{n n^{\prime}}^{-1}=1 / \bar{m}_{0}^{2}$. Now insert the above expression for $\phi_{n}$ in equation (11) ${ }^{4}$. The term proportional to $(1 / 2-h) / \kappa \bar{m}_{0}^{2}$ cancels the $\ln g$ term of Eq. (11). Averaging over the thermal fluctuations the resulting expression for $\epsilon$, we are led to compute $\left\langle\rho_{n} \partial \mathcal{S} / \partial \phi_{n^{\prime}}\right\rangle$, which can be written as

$$
\left\langle\rho_{n} \frac{\partial \mathcal{S}}{\partial \phi_{n^{\prime}}}\right\rangle=-\frac{1}{\mathcal{Z}} \int[d \phi] \rho_{n} \frac{\partial}{\partial \phi_{n^{\prime}}} \mathrm{e}^{-\mathcal{S}}
$$

Integrating by parts and taking into account that $\partial \rho_{n} / \partial \phi_{n^{\prime}}=\delta_{n n^{\prime}} \rho_{n}\left(1-\rho_{n}\right)$, we get

$$
\left\langle\rho_{n} \frac{\partial \mathcal{S}}{\partial \phi_{n^{\prime}}}\right\rangle=\delta_{n n^{\prime}}\left\langle\rho_{n}\left(1-\rho_{n}\right)\right\rangle
$$

The term linear in $\rho_{n}$ cancels the term proportional to $\kappa^{-1} \overline{\mathcal{D}}_{n n}^{-1}$ of the averaged Eq. (111). Hence, we obtain for the averaged energy

$$
\epsilon=k_{B} T\left[\frac{3}{2} \rho-\frac{1}{2} \frac{1}{V} \sum_{n n^{\prime}} \kappa^{-1}\left\langle\rho_{n} \overline{\mathcal{D}}_{n n^{\prime}}^{-1} \rho_{n^{\prime}}\right\rangle+\frac{1}{2} \frac{1}{V} \sum_{n} \kappa^{-1}\left\langle\rho_{n} \overline{\mathcal{D}}_{n n}^{-1} \rho_{n}\right\rangle\right] .
$$

It suffices to write $n^{\prime}=n+r$ to realize that the above equation is Eq. (12).

\section{References}

[1] P.J.E. Peebles, Principles of Physical Cosmology (Princeton University Press, 1993).

[2] U. Lindner, M. Einasto, J. Einasto, W. Freudling, K. Fricke, V. Lipovetsky, S. Pustilnik, Y. Izotov, and G. Richter, Astron. Astrophys. 314, 1 (1996).

[3] J. Einasto, astro-ph/0011334.

[4] P.J.E. Peebles, Ap. J. 557, 495 (2001).

[5] F. Sylos Labini, M. Montuori, and L. Pietronero, Phys. Rep. 293, 61 (1998).

\footnotetext{
${ }^{4}$ Not in the term $\rho_{n}=\mathrm{e}^{\phi_{n}} /\left(1+\mathrm{e}^{\phi_{n}}\right)$.
} 
[6] M. Davis, in Critical Dialogues in Cosmology, Ed. N. Turok, (World Scientific, Singapore, 1997).

[7] P.J.E. Peebles, in Les Rencontrees de Physique de la Vallee d'Aosta, Ed. M. Greco (1998).

[8] L. Guzzo, New Astronomy 2, 517 (1997).

[9] R.B. Larson, Mont. Not. R Astron. Soc. 194, 809 (1981).

[10] A. Hetem jr. and J.R.D. Lepin, Astron. Astrophys. 270, 451 (1993).

[11] C. Kramer et al. Astron. Astrophys. 329, 249 (1998).

[12] J. Stutzki et al. Astron. Astrophys. 336, 697 (1998).

[13] H.J. De Vega, N. Sánchez, and F. Combes, Nature 383, 53 (1996); Phys. Rev. D54, 6008 (1996).

[14] T. Goldman, D. Hochberg, R. Laflamme, and J. Pérez-Mercader, Phys. Lett. A222, 177 (1996).

[15] W. C. Saslaw, Gravitational physics of stellar and galacti systems (Cambridge University Press, 1987).

[16] J. Frölich, in Renormalization Theory, Eds. G. Velo and A.S. Wightman (Reidel, Dordrech/Boston, 1976).

[17] A.M. Polyakov, Nucl. Phys. B20, 429 (1977).

[18] S. Samuel, Phys. Rev. D18, 1916 (1978).

[19] T. Padmanabham, Phys. Rep. 188, 285 (1990).

[20] E. Follana and V. Laliena, Phys. Rev. E61, 6270 (2000).

[21] D.H.E. Gross, Microcanonical Thermodynamics (World Scientific, 2001).

[22] D. Lynden-Bell, in Statistical Physics, Eds. A. Gervais, D. Iagolnitzer, M. Moreau, and Y. Pomeau (North-Holland, 1999).

[23] P. Hertel and W. Thirring, Ann. Phys. (N.Y.) 63, 520 (1971).

[24] B. Stahl, M.K.-H. Kiessling, and K. Schindler, Planet. Space Sci. 43, 271 (1995).

[25] A. Torcini and M. Antoni, Phys. Rev. E59, 2746 (1999).

[26] P.-H. Chavanis and J. Sommeria, Mon. Not. R. Astr. Soc. 296, 569 (1998).

[27] V.P. Youngkins and B.N. Miller, Phys. Rev. E62, 4583 (2000).

[28] I. Ispolatov and E.G.D. Cohen, Phys. Rev. E64, 056103 (2001).

[29] P.-H. Chavanis, Phys. Rev. E65, 056123 (2002). 
[30] P.-H. Chavanis and I. Ispolatov, Phys. Rev. E66, 036109 (2002).

[31] H.J. De Vega and N. Sánchez, Phys. Lett. B490, 180 (2000); Nucl. Phys. B625, 409 (2002); ibid 460 .

[32] V. Laliena, Nucl. Phys. B668, 403 (2003); astro-ph/0309225.

[33] V. Laliena, Phys. Rev. E59, 4786 (1999).

[34] R. Emden, Gaskugeln (Druck und Verlag von B.G. Teubner, Leipzig, 1907).

[35] S. Chandrasekhar, Stellar Structure (Chicago University Press, 1939).

[36] V.A. Antonov, Vest. leningr. gos. Univ. 7, 135 (1962).

[37] D. Lynden-Bell and R. Wood, Mon. Not. R. astr. Soc. 138, 495 (1968).

[38] J. Gaite, A. Domínguez, and J. Pérez-Mercader, Astrophys. J. 522, L5 (1999).

[39] E. Abdalla and M. Rezha Rahimi Tabar, Phys. Lett. B440, 339 (1998). 